\title{
Contact Stability on Cutting Characteristics of Polycarbonate Sheets Subjected to Two-Line Wedge Indentation*
}

\author{
Shigeru NAGASAWA**, Takuya TAKAHASHI** and Masatoshi FUJIKURA** \\ ** Department of mechanical engineering, Nagaoka University of Technology \\ 1603-1 Kamitomioka, Nagaoka, Niigata 940-2188, Japan \\ E-mail: snaga@mech.nagaokaut.ac.jp (Shigeru Nagasawa)
}

\begin{abstract}
This paper describes a pushing cut process of a piled-up polycarbonate (PC) sheet. Resin laminated sheets such as thermal insulation films or liquid crystal panel are recently processed by using a wedge indentation due to its production efficiency and its precision cutting. However, deformation of laminated resin sheet is often unstable during cutting or creasing process, and its dynamic behavior is not sufficiently revealed. In this work, the cutting line force of several center bevel blades was measured by load cells, and the cutting deformation of the PC sheet was observed by a CCD camera in order to reveal the effect of blade tip profile and the contact condition. The deformation flow in the side view of the PC sheet was observed with respect to the blade indentation by varying the blade tip angle, the feed velocity and the surface contact condition.
\end{abstract}

Key words: Shear, Cutting, Sticking, Friction, Facets, Apex Angle, Adhesion

\section{Introduction}

A wedge indentation processing is widely used for cutting off a complicated-formed pattern from a sheet material such as carton boxes, labels, insulation films and similar metal thin sheets. As the LCD panels or similar laminated resin sheets (transparent panels) can be also processed with a wedge blade (e.g., Pinnacle die, Thomson knife) by an underlay mounted on a flat counter plate, this processing method becomes recently important for high quality production and to realize the fundamental cutting characteristics of the worksheet is required for utilizing this processing method.

As one of similar processing, there is a converting industry of white-coated paperboard for packages or boxboards ${ }^{(1)(2)(3)}$. The cutting mechanics of laminated resin sheets and that of paperboards are supposed to be similar with each other from the physical aspects of laminated inner layers, and material flow around a cutting edge. The resin materials can be relatively seen as the isotropic properties compared with paperboards, while adhesive tapes (glues) are additional substrates which change the frictional condition or the wetness of blade surface. There are many complicated behaviors unsolved during the resin sheet cutting depending on its production purpose. Theoretical works ${ }^{(4),(5)}$ and experimental material flow observation of ductile metal ${ }^{(6)(7)(8)}$ on wedge indentation into semi-infinite isotropic plastic body are useful to understand the breaking pattern, the influence of friction, the tip angle of blade, the worksheet thickness and the yield stress of worksheet with respect to the indentation force. Regarding the resin sheet cutting, Masumori et al investigated the circular die-punch shearing of Polyvinyl chloride (PVC), polycarbonate(PC) and polypropylene (PP) ${ }^{(9)}$. Maeda et al investigated the effect of flexible underlay on punching of PC, PVC and so on by a knife-edge tool ${ }^{(10)}$. Those researches were based on the 
punch-die shearing mechanics. However, the traditional models were not sufficient to explain the cutting load characteristics and the deformation behavior of the piled-up resin worksheets during the facets center bevel blade indentation process, especially for the stability of interface sticking/sliding phenomena. Deformation of laminated resin sheet is often unstable during cutting or creasing process ${ }^{(11)}$, and the dynamic behavior is not sufficiently revealed.

In this paper, a pushing shear test of a couple of facets blade on two pieces of piled-up polycarbonate (PC) sheets of $t=0.5 \mathrm{~mm}$ thickness was experimentally carried out by varying the blade tip angle, by considering the glue-coated layer of the PC sheet and by adding an adhesive tape of $t_{\mathrm{g}}=50 \mu \mathrm{m}$ thickness as the intermediate layer, in order to reveal the effect of tip profile of two-line (facets) blade, the frictional restriction among the blade tip and two pieces of piled-up PC sheets, the underlay effect of the lower worksheet on the deformation flow of the upper worksheet.

\section{Experimental condition}

Fig.1 illustrates the experimental setup for a blade pushing into a piled-up specimen of two pieces of polycarbonate (PC) sheet. On the experimental apparatus, the upper crosshead moved downward with a feed velocity $V$, which was chosen as 0.05 and $0.5 \mathrm{~mm} \cdot \mathrm{s}^{-1}$. The upper crosshead had a facets blade mounted on a load cell with the maximum load of 10 $\mathrm{kN}$. The piled-up PC sheets (worksheets) were placed on a hard counter plate (Japan Industrial Standard (JIS): SUS630 with the average hardness of $510 \mathrm{HV}$, the surface roughness $R_{a}=0.2 \mu \mathrm{m}$ ) which was fixed on the lower crosshead.

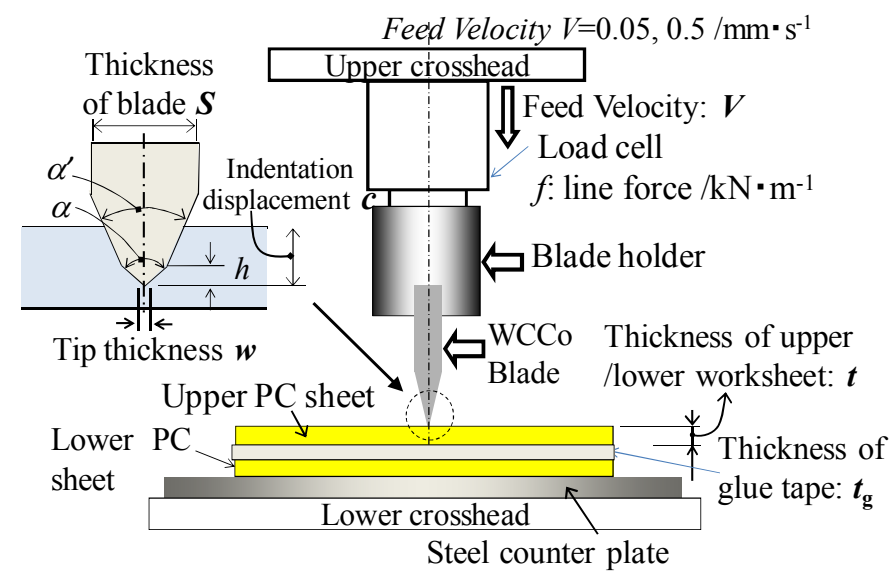

Fig.1 Schematic of experimental apparatus

As the PC sheet was initially coated with a water-soluble glue layer which was used for attaching a masking film, all the specimens of PC sheet were prepared with two conditions: (i) a half of all the specimens were sufficiently washed and dried, (ii) the others were used without cleaning. This surface condition was discussed with respect to the friction effect. In case of washed surface condition (i), the specimens were furthermore prepared for two kinds of combination: (a) two pieces of worksheet piled-up without any adhesive tape, (b) two pieces of worksheet attached with an acrylic based adhesive tape (DF9100) of $t_{\mathrm{g}}=50 \mu \mathrm{m}$ thickness. In case of unwashed condition (ii), only the piled-up condition without any adhesive tape (a) was considered. Table $\mathbf{1}$ shows the In-plane mechanical properties of polycarbonate (PC) sheet in Machine direction (MD) under the strain rate of $0.83 \times 10^{-3} \mathrm{~s}^{-1}$. The adhesive tape (DF9100) had the Out-Of-Plane Young's modulus of $4.0(2.4 \sim 5.0) \mathrm{MPa}$ for the compressive true strain of $0.1 \sim 0.2$ under the strain rate of $8.3 \times 10^{-3} \mathrm{~s}^{-1}$.

Each PC sheet of $0.5 \mathrm{~mm}$ thickness was a rectangle sheet with the length of $l=40 \mathrm{~mm}$ and the width of $b=20 \mathrm{~mm}$. The position of cutting blade was vertical to the specimen, while 
the angle $\phi$ of cutting line direction with respect to MD of the PC sheet was chosen as $\phi=$ $90^{\circ}$.

The facets cutting blade, which was made with Cemented Carbide (W-C-Co, FM10K), had the length of $L=30 \mathrm{~mm}$, the height of $H=9 \mathrm{~mm}$, the thickness of $S=0.90 \mathrm{~mm}$, and the initial tip thickness of $w=0.8 \mu \mathrm{m}$ in average. The apex angle $\alpha$ of the first facet was chosen as $90^{\circ}$ and the surface roughness was $R_{a}=0.01 \mu \mathrm{m}$ (on grinding surface). The height of the first facet $h$ was $150 \mu \mathrm{m}(h / t=0.3)$, while the secondary bevel angle $\alpha^{\prime}$ was chosen as $30^{\circ}$.

Table 1 In-plane mechanical properties of polycarbonate (MD)

\begin{tabular}{|c|c|c|c|}
\hline $\begin{array}{c}\text { Young's Modu. } \\
E / \mathrm{GPa}\end{array}$ & $\begin{array}{c}\text { Proof Str. } \\
\sigma_{Y} / \mathrm{MPa}\end{array}$ & $\begin{array}{c}\text { Tens. Str. } \\
\sigma_{B} / \mathrm{MPa}\end{array}$ & $\begin{array}{c}\text { Breaking Strain } \\
\varepsilon_{B}\end{array}$ \\
\hline 2.5 & 61.8 & 70.6 & 1.28 \\
\hline
\end{tabular}

The facets cutting blade, which was made with Cemented Carbide (W-C-Co, FM10K), had the length of $L=30 \mathrm{~mm}$, the height of $H=9 \mathrm{~mm}$, the thickness of $S=0.90 \mathrm{~mm}$, and the initial tip thickness of $w=0.8 \mu \mathrm{m}$ in average. The apex angle $\alpha$ of the first facet was chosen as $64^{\circ}$ and $90^{\circ}$, and the surface roughness was $R_{a}=0.01 \mu \mathrm{m}$ (on grinding surface). The height of the first facet $h$ was $150 \mu \mathrm{m}(h / t=0.3)$, while the secondary bevel angle $\alpha^{\prime}$ was chosen as $30^{\circ}$.

The three kinds of friction coefficient $\mu_{C}, \mu_{\mathrm{U}}$ and $\mu_{\mathrm{P}}$ were experimentally measured by the horizontal method based on JIS-P8147, and shown in Table 2. Here, they were measured with the applied contact pressure $p=6 \mathrm{kPa}$ between the cutting blade, the PC sheet and the counter plate, respectively. When the applied contact pressure was increased with the PC sheet, those friction coefficients were empirically increased, while the washed surface was apt to be slippery. In case of washed condition with the PC sheets, the $\mu_{\mathrm{U}}$ was roughly 0.1 for $p=10 \mathrm{kPa}$, the $\mu_{\mathrm{C}}$ was larger than 0.4 for $p>15 \mathrm{kPa}$, and the $\mu_{\mathrm{P}}$ was larger than 0.15 for $p>15 \mathrm{kPa}$, respectively.

Table 2 Friction coefficients measured by the horizontal method $(6 \mathrm{kPa})$

\begin{tabular}{|l|c|c|c|}
\hline $\begin{array}{c}\text { Symbol } \\
\text { (contact surface) }\end{array}$ & $\begin{array}{c}\mu_{\mathrm{C}} \\
\text { (Blade-PC) }\end{array}$ & $\begin{array}{c}\mu_{\mathrm{U}} \\
\text { (PC-PC) }\end{array}$ & $\begin{array}{c}\mu_{\mathrm{P}} \\
\text { (PC-Counter plate) }\end{array}$ \\
\hline (i) Washed & $0.06(0.05 \sim 0.08)$ & $0.04(0.03 \sim 0.06)$ & $0.06(0.05 \sim 0.11)$ \\
\hline (ii) Unwashed & $0.40(0.38 \sim 0.42)$ & $0.49(0.44 \sim 0.60)$ & $0.30(0.20 \sim 0.40)$ \\
\hline
\end{tabular}

The applied pushing force $F$ was measured by the load cell. The indentation displacement of the cutting blade into the PC sheet $c$ was measured as the upper crosshead displacement until the time when the upper worksheet was completely separated (almost for $c / t<1.5) . f(=F / b) \mathrm{kN} \cdot \mathrm{m}^{-1}$ is the line force applied in vertical to the cutting blade. In order to observe the deformation flow of the side view of PC sheet during the blade indentation, CCD microscope was used for recording the video image data. The experiment was carried out under the following conditions; room temperature: $297 \mathrm{~K}$, room humidity: $50 \%$, number of samples: twenty pieces for each condition.

\section{Results and discussion}

\subsection{Comparison of washed surface with unwashed surface}

In order to know the fundamental cutting behavior of two pieces of piled-up PC sheet without any adhesive tape, the $\alpha^{\prime} / \alpha=30 / 64^{\circ}$ and/or $30 / 90^{\circ}$ facets blade was indented to the upper PC sheet with the feed velocity of $V=0.05 \mathrm{~mm} \cdot \mathrm{s}^{-1}$. The deformation flow of the upper PC sheet was recorded by using CCD microscope and its line force was measured by the load cell.

Fig.2 shows a couple of representative video photographs as side views of upper PC sheet which was going to be cut off by using the $30 / 90^{\circ}$ facets blade. The unwashed specimens were shown in Fig.2(a) and the washed specimens were shown in Fig.2(b). The 
washed surface is corresponded to a slippery state, while the unwashed surface is to be a sticky state. The corresponded load responses were shown in Fig.3. Here, the blade indentation was programmed in a digital press system with the specified feed velocity up to $c / t=1.6$ or $2.0(t=0.5 \mathrm{~mm})$. The value of $n_{\mathrm{C}}$ was the sequential number of a specimen picked up from 20 samples.

Through those photographs and cutting line force responses of the $30 / 90^{\circ}$ facets blade several features were found:

(1) In case of unwashed surface condition (sticky state), there is remarkable dispersion or different response of the cutting line force for $c / t>0.5$, while the dispersion is generally small except for the final breaking of the upper PC sheet in case of washed surface condition (slippery state). Since there was dispersion of the peak cutting line force in both cases, we statistically detected there were the quasi-lower bound peak line force and the quasi-upper bound peak line force.

(2) In case of unwashed surface condition, when the final breaking of the upper PC sheet occurred at near $c / t=1$, the deformation (dent) of the lower PC sheet was relatively small, while when the peak cutting line force relatively increased, the peak position was apt to be postponed and the lower PC sheet was dent in the thickness direction due to a high pressure from the upper PC sheet. This tendency of branching deformation was disappeared and the lower PC sheet was hardly dent before cutting off the upper PC sheet, in the case of washed surface condition.
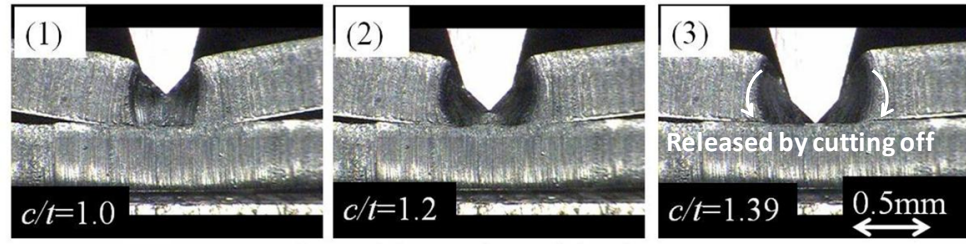

In case of quasi-lower bound load response $\left(n_{C}=11\right)$
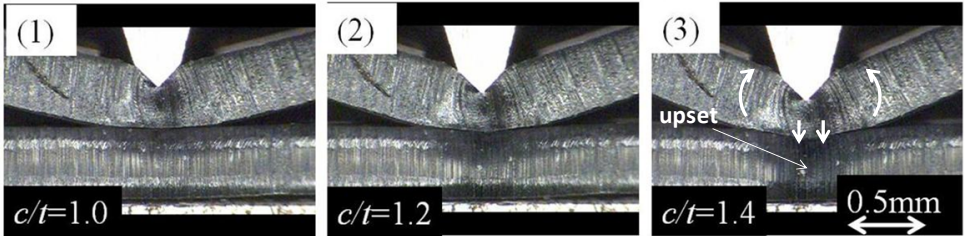

In case of quasi-upper bound load response $\left(n_{C}=17\right)$

(a) In case of sticky (unwashed) surface w/o adhesive tape
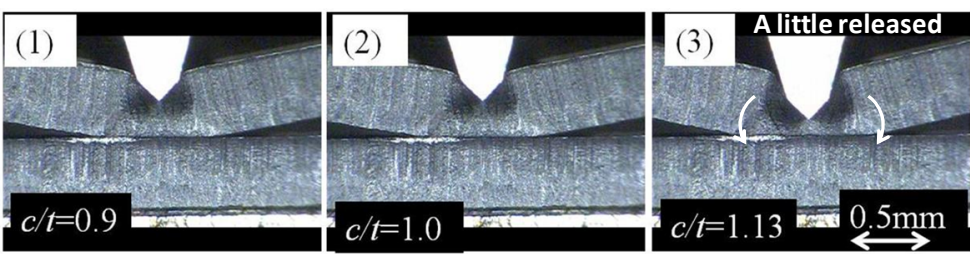

In case of quasi-lower bound load response $\left(n_{\mathrm{C}}=16\right)$

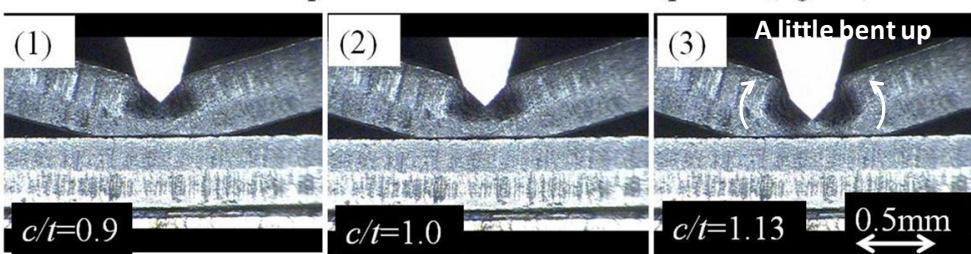

In case of quasi-upper bound load response $\left(n_{\mathrm{C}}=3\right)$

(b) In case of slippery (washed) surface w/o adhesive tape

Fig.2 CCD side-views of PC sheets sheared by $30 / 90^{\circ}$ facets blades

$$
\left(V=0.05 \mathrm{~mm} \cdot \mathrm{s}^{-1}, t=0.5 \mathrm{~mm}\right)
$$

(3) In case of the quasi-upper bound condition of Fig.2(a), the secondary bevel of $\alpha^{\prime}=30^{\circ}$ 
had almost detached from the upper surface of the upper PC sheet during the blade indentation, except for a neighborhood region of the end point of the first facet. In other cases of the quasi-lower bound condition of Fig.2(a), the quasi-upper bound condition of Fig.2(b) and the quasi-lower bound condition of Fig.2(b), the secondary bevel begun to sink and attached to the upper surface of the upper PC sheet for $c / t>1.0 \sim 1.2$. Namely, it seems that the early peak point of load response (at $c / t=1.0 \sim 1.2$ ) corresponds to the surface breaking and the sliding deformation on the first bevel of $\alpha=90^{\circ}$. As the result of this surface breaking, the interface between the upper PC and the lower PC sheet was supposed to become slippery.

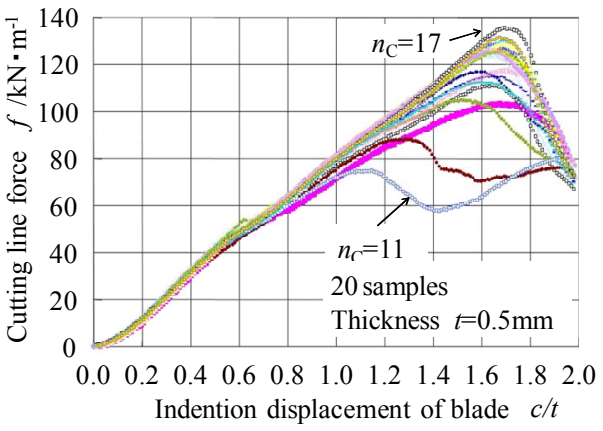

(a) Unwashed specimens

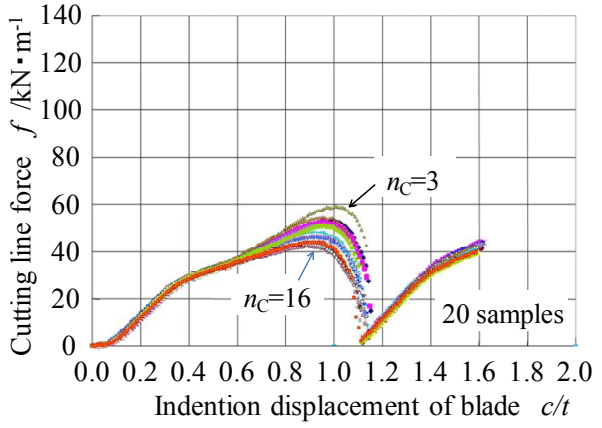

(b) Washed specimens

Fig.3 Relationship between cutting line force and blade indentation displacement in case of $V=0.05 \mathrm{~mm} / \mathrm{s}, \alpha^{\prime} / \alpha=30 / 90^{\circ} \mathrm{w} / \mathrm{o}$ adhesive tape
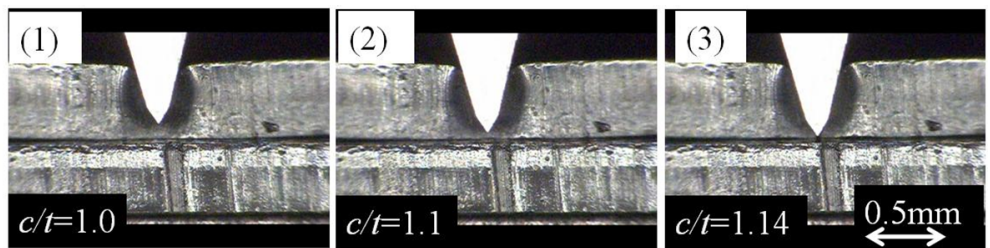

In case of quasi-lower bound load response $\left(n_{\mathrm{C}}=7\right)$
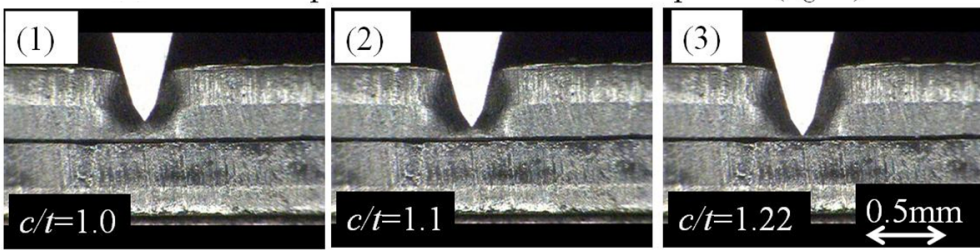

In case of quasi-upper bound load response $\left(n_{\mathrm{C}}=9\right)$

(a) In case of sticky (unwashed) surface w/o adhesive tape
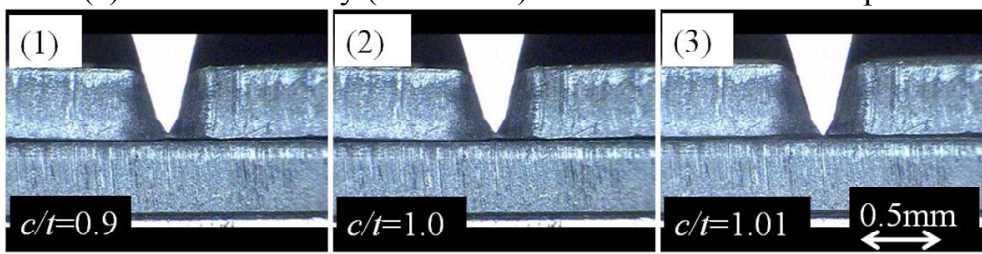

In case of quasi-lower bound load response $\left(n_{C}=20\right)$
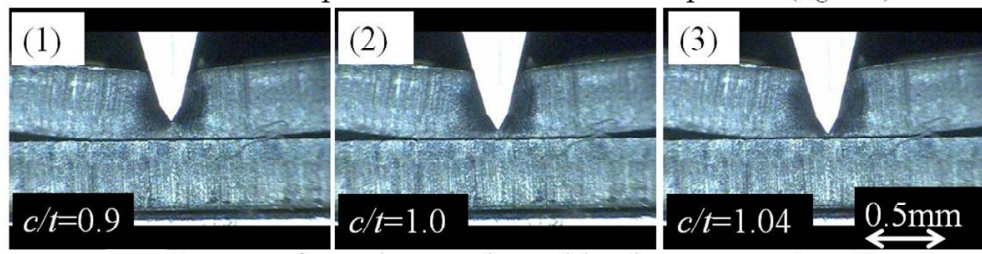

In case of quasi-upper bound load response $\left(n_{C}=3\right)$

(b) In case of slippery (washed) surface w/o adhesive tape

Fig.4 CCD side-views of PC sheets sheared by $30 / 64^{\circ}$ facets blades

$$
\left(V=0.05 \mathrm{~mm} \cdot \mathrm{s}^{-1}, t=0.5 \mathrm{~mm}\right)
$$


(4) When the indentation displacement of blade $c / t$ exceeded about 0.6 , the upper PC sheet was apt to be bent up and the backside of the upper PC sheet was detached from the lower PC sheet at a distance of $2 t$ or the similar length from the blade center position. It seems that the lateral component of wedging force causes the lateral (in-plane) elongation flow as camber of the upper PC sheet and the inflection load point at near $c / t=0.4 \sim 0.6$.

(5) When we replaced the piled-up PC sheets of $0.5 \mathrm{~mm}$ to another PC sheet of $1.0 \mathrm{~mm}$ as a trial working, the load response was quite similar to the case of $n_{\mathrm{C}}=17$ (Fig.3(a)). Hence, it seems that the quasi-upper bound load response of the $30 / 90^{\circ}$ blade is same as a fixing mode of the two pieces of PC sheets without any bent-up and any partial detaching.

Next, another facets blade of $30 / 64^{\circ}$ was used for the pushing shear test of the piled-up PC sheet without adhesive tape, in order to discuss with the effect of apex angle. Fig.4(a) shows a couple of video photographs of the PC sheet sheared by the $30 / 64^{\circ}$ facets in case of unwashed surface condition, while Fig.4(b) shows that in case of washed surface condition. The corresponded load responses were shown in Fig.5(a),(b) respectively $\left(V=0.05 \mathrm{~mm} \cdot \mathrm{s}^{-1}\right)$.

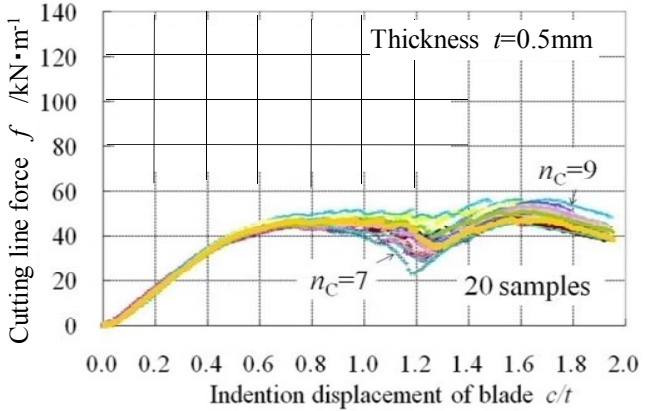

(a) Unwashed surface

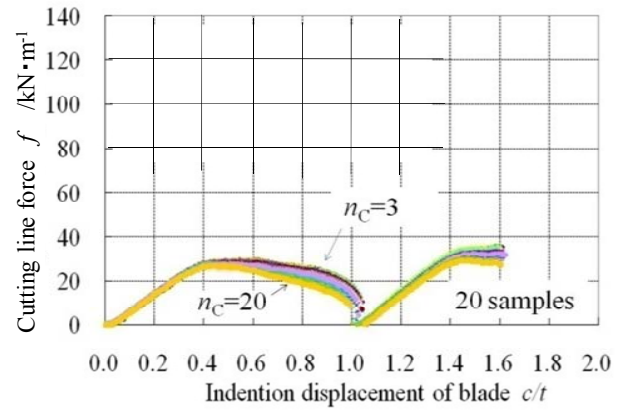

(b) Washed surface

Fig.5 Relationship between cutting line force and blade indentation displacement in case of $V=0.05 \mathrm{~mm} \cdot \mathrm{s}^{-1}$ and $\alpha^{\prime} / \alpha=30 / 64^{\circ} \mathrm{w} / \mathrm{o}$ adhesive tape

From Fig.4 and Fig.5, the following features (6) (9) were detected.

(6) Comparing $n_{\mathrm{C}}=20$ with $n_{\mathrm{C}}=3$ in Fig.4(b) and Fig.5(b), there was locally a necking deformation beneath the blade tip and the bent-up angle of the upper PC sheet was quite small in case of the quasi-lower bound load response, while any necking (relief) was not detected beneath the blade tip and the bent-up angle was relatively large in case of the quasi-upper bound load response. Those tendencies of bent-up angle were basically same as the case of $30 / 90^{\circ}$ blade.

(7) The dispersion of load response and the branching of bent-up deformation were detected although the variance of those tendencies was relatively small. The dispersion of load response specified as transition between the quasi-lower bound and the quasi-upper bound load response was mainly observed during the latter half $(c / t>0.6)$. The fixing mode which was seen in case of the $30 / 90^{\circ}$ blade between 2 pieces of PC sheets was not clearly detected. This was supposed to be caused by the secondary bevel indentation for $c / t>0.6$.

(8) As a trial cutting, we have investigated the relationship between the cutting line force and the indentation displacement of blade by using one-line center bevel blade of $\alpha=\alpha^{\prime}=$ $30^{\circ}$ and $/$ or $60^{\circ}$ in case of washed surface condition. From this result, it was revealed that the early load response on $c / t=0 \sim 0.4$ was decided by the first bevel angle $\alpha$, while the latter half of the load response $(c / t=0.6 \sim 1.0)$ was basically caused by the secondary bevel angle $\alpha^{\prime}$.

(9) Regarding the unwashed condition, we found that the item (1) of $30 / 90^{\circ}$ blade was applicable to the case of $30 / 64^{\circ}$ blade.

\subsection{Effect of feed velocity on cutting load response}

Fig.6 shows the load response for $V=0.5 \mathrm{~mm} \cdot \mathrm{s}^{-1}$. In this case, the cutting deformation 
and the load response were almost similar to that of $V=0.05 \mathrm{~mm} \cdot \mathrm{s}^{-1}$ (Fig.3), except for the following several features. The peak cutting line force of the former $\left(0.5 \mathrm{~mm} \cdot \mathrm{s}^{-1}\right)$ was apt to be larger than that of the latter $\left(0.05 \mathrm{~mm} \cdot \mathrm{s}^{-1}\right)$.

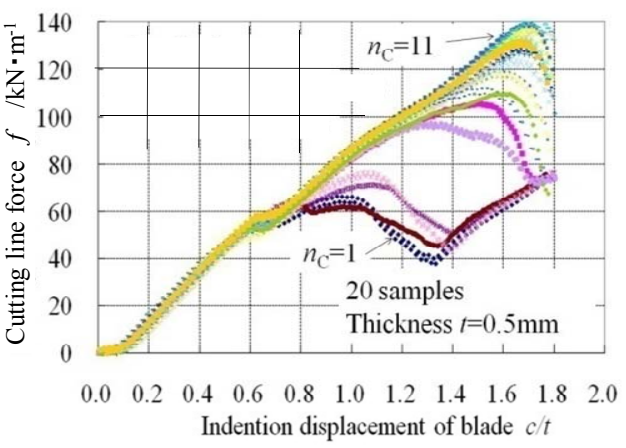

(a) Unwashed surface

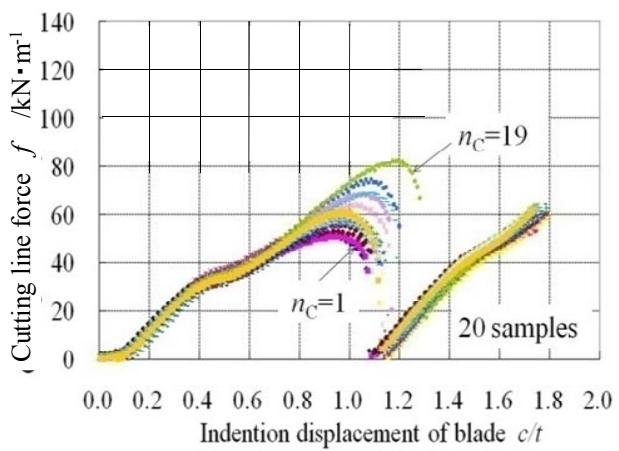

(b) Washed surface

Fig.6 Relationship between cutting line force and blade indentation displacement in case of $V=0.5 \mathrm{~mm} / \mathrm{s}$ and $\alpha^{\prime} / \alpha=30 / 90^{\circ} \mathrm{w} / \mathrm{o}$ adhesive tape

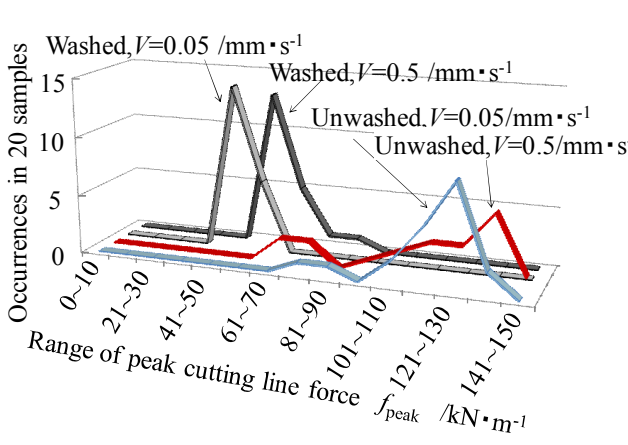

(a) In case of $30 / 90^{\circ}$ blade

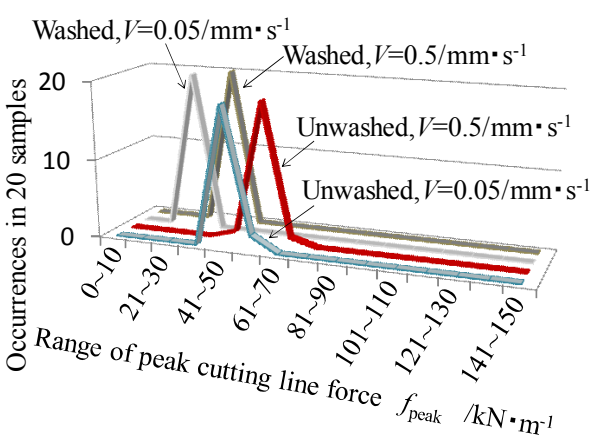

(b) In case of $30 / 64^{\circ}$ blade

Fig.7 Histogram of peak cutting line force occurrences w/o adhesive tape

Regarding the effects of surface friction in washed and unwashed condition and the effect of feed velocity $V$, the peak cutting line force $f_{\text {peak }}$ was arranged with $10 \mathrm{kN} \cdot \mathrm{m}^{-1}$ of incremental range from the aspects of statistical occurrences as shown in Fig.7(a),(b). From those graphs, it is confirmed that the higher speed is apt to increase $f_{\text {peak }}$, and the numbers of most active range on $\alpha=90^{\circ}$ was apt to be larger than that on $\alpha=64^{\circ}$. The washed surface condition (slippery state) decreases $f_{\text {peak }}$ and also reduces its dispersion.

\subsection{Effects of adhesive tape on cutting response of upper worksheet}

Fig.8 shows a couple of representative video photographs of the attached PC sheets with an acrylic based adhesive tape of $50 \mu \mathrm{m}$ during the cutting test by the $30 / 90^{\circ}$ facets blade. Here, the PC sheets were sufficiently washed and dried before setting up, and the feed velocity was chosen as $V=0.05 \mathrm{~mm} \cdot \mathrm{s}^{-1}$. The blade surface was carefully cleaned with a liquid alcohol before every cutting. As shown in those figures, the left side wedge contact sometimes (for a certain short time) differed from the right side wedge contact with the sheared surface.

Fig.9 (a) shows the cutting line force response for $V=0.05 \mathrm{~mm} \cdot \mathrm{s}^{-1}$ (corresponded to Fig. 8) and Fig. 9 (b) is that for $V=0.5 \mathrm{~mm} \cdot \mathrm{s}^{-1}$. Here, the video photographs of deformation flow of PC sheets $\left(V=0.5 \mathrm{~mm} \cdot \mathrm{s}^{-1}\right)$ were macroscopically similar to that of $V=0.05 \mathrm{~mm} \cdot \mathrm{s}^{-1}$, while the viscosity effect of adhesives seemed to increase the cutting resistance. From Fig.9, the quasi-stationary response which occurred after passing through the peak cutting line force was detected for $c / t=1.0 \sim 1.1$. Like this quasi-stationary response was seen also in Fig.3(a) $\left[n_{\mathrm{C}}=11\right]$ and Fig.6(a) $\left[n_{\mathrm{C}}=1\right]$. 


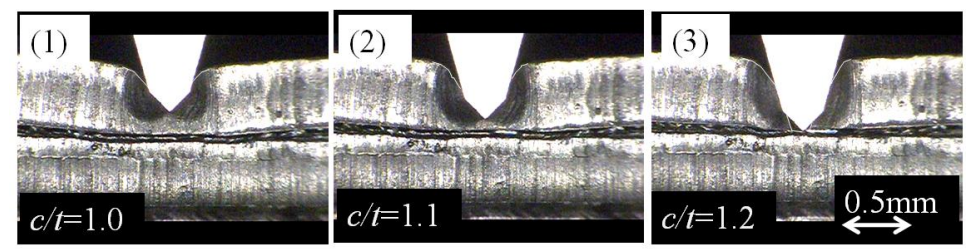

(a) In case of quasi-lower bound load response $\left(n_{\mathrm{C}}=6\right)$

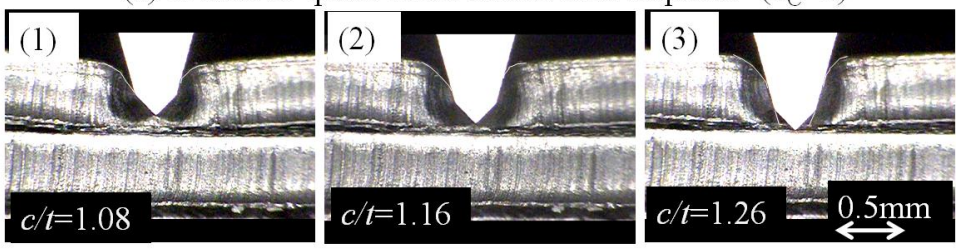

(b) In case of quasi-upper bound load response $\left(n_{\mathrm{C}}=8\right)$

Fig.8 CCD side views of PC sheet sheared by $30 / 90^{\circ}$ facets $\left(V=0.05 \mathrm{~mm} \cdot \mathrm{s}^{-1}, t=0.5 \mathrm{~mm}\right.$, washed surface with adhesive tape )

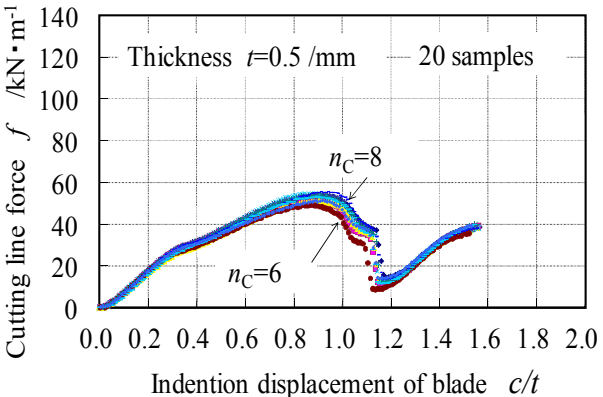

(a) $\quad V=0.05 \mathrm{~mm} \cdot \mathrm{s}^{-1}$

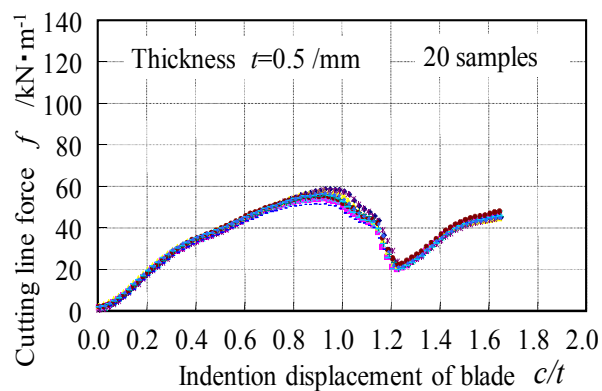

(b) $V=0.5 \mathrm{~mm} \cdot \mathrm{s}^{-1}$

Fig.9 Relationship between cutting line force and blade indentation displacement in case of $\alpha^{\prime} / \alpha=30 / 90^{\circ}$ on washed surface with adhesive tape

From the aspect of geometrical condition observed through the video movies, the quasi-stationary response is supposed to correspond with the secondary bevel indentation. Comparing Fig.3(b) with Fig.9(a), it is found that the dispersion of $f_{\text {peak }}$ was relatively reduced when the adhesive tape was inserted between two pieces of PC. This tendency was also seen in the pair of Fig.6(b) and Fig.9(b). Therefore, the sliding state between the upper PC sheet and the lower PC sheet seems to be the primary factor for controlling the dispersion behavior at near the peak point of $f_{\text {peak }}$.

In case of the $30 / 64^{\circ}$ facets blade indentation, as the 20 samples of the load response were relatively stable for both the feed velocity 0.05 and $0.5 \mathrm{~mm} \cdot \mathrm{s}^{-1}$, there was not any large dispersion of $f_{\text {peak }}$.

\subsection{Sheared profile of upper worksheet}

Fig.10 shows side views of a couple of sheared section by using the $30 / 90^{\circ}$ facets blade. Fig.10 (a) shows CCD photographs in case of unwashed specimens with the feed velocity of $V=0.05 \mathrm{~mm} \cdot \mathrm{s}^{-1}$, while Fig. 10 (b) shows that in case of washed specimens with $V=0.5 \mathrm{~mm} \cdot \mathrm{s}^{-1}$. It is found that a sheared profile on the quasi-upper bound load response is apt to be different from that on the quasi-lower bound. By measuring the inclined angle $\beta$, the elevation angle $\gamma$, and the necked height $h_{\mathrm{n}}$ as shown in Fig.11, the sheared profile was classified into three modes A, B and C. As the load response is varied with the friction of contact surfaces, the feed velocity and the bevel angle, the sheared profile of PC sheet seems to be also affected by those parameters.

Fig.12 shows the inclined angle $\beta$ in the three cases of (a) unwashed surface without adhesive tape, (b) washed surface without adhesive tape and (c) washed surface with adhesive tape (GT). Where, $\mathrm{C} 1, \mathrm{C} 2, \mathrm{C} 3$, and $\mathrm{C} 4$ denote the feed velocity and the blade tip 
angles. In Fig.12, the error bars mean the maximum and the minimum value occurred. In the cases of $\mathrm{C} 1, \mathrm{C} 2$ with unwashed surface of specimens, the dispersion of $\beta$ was large and roughly from 15 to $45^{\circ}$. They seemed to be derived from a half angle of $\alpha^{\prime} / \alpha=30 / 90^{\circ}$.

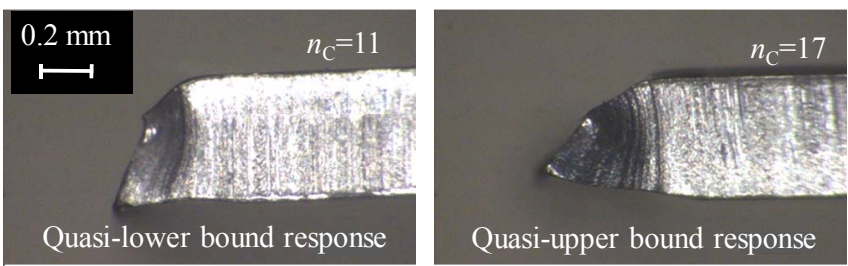

(a) Unwashed, $V=0.05 / \mathrm{mm} \cdot \mathrm{s}^{-1}$
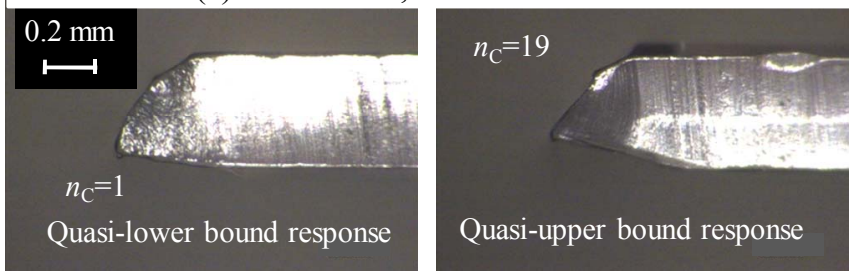

(b) Washed, $V=0.5 / \mathrm{mm} \cdot \mathrm{s}^{-1}$

Fig.10 Side views of sheared section by CCD microscope $\left(\alpha^{\prime} / \alpha=30 / 90^{\circ}\right)$

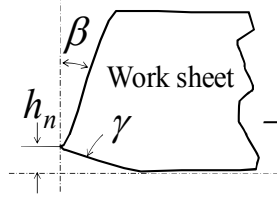

(a) Mode A

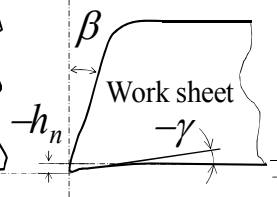

(b) Mode B

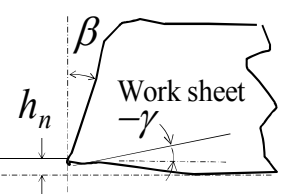

(c) Mode C

Fig.11 Definition of inclined angle, elevation angle and necked height for three modes

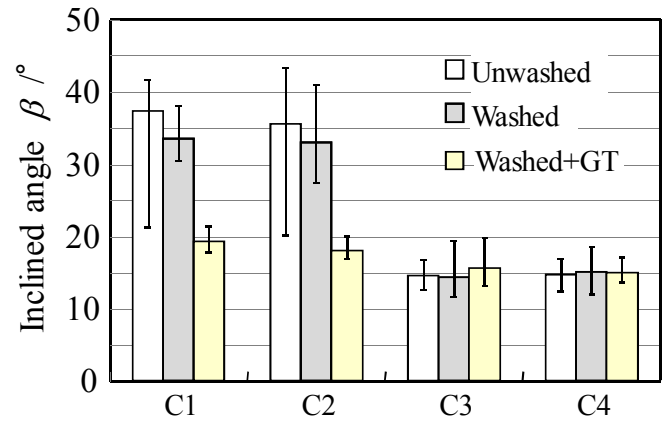

$$
\begin{aligned}
& \mathrm{C} 1: \alpha^{\prime} / \alpha=30 / 90^{\circ}, \\
& V=0.05 / \mathrm{mm}^{\circ} \mathrm{s}^{-1} \\
& \mathrm{C} 2: \alpha^{\prime} / \alpha=30 / 90^{\circ}, \\
& V=0.5 / \mathrm{mm}^{\cdot} \mathrm{s}^{-1} \\
& \mathrm{C} 3: \alpha^{\prime} / \alpha=30 / 64^{\circ}, \\
& V=0.05 / \mathrm{mm} \cdot \mathrm{s}^{-1} \\
& \mathrm{C} 4: \alpha^{\prime} / \alpha=30 / 64^{\circ}, \\
& V=0.5 / \mathrm{mm}^{\circ} \cdot \mathrm{s}^{-1}
\end{aligned}
$$

Fig. 12 Comparison of inclined angle w.r.t. adhesive tape, feed velocity and tip angle

In case of washed surface of specimens, the dispersion of $\beta$ was relatively small. This tendency matches that the load response was almost stable and the position of $f_{\text {peak }}$ occurred at $c / t=1.0 \sim 1.2$.

When the adhesive tape was inserted to the specimens, as the value of $\beta$ was settled down without any large disparsion, it seemed that the sliding friction resistance between the upper PC sheet and the underlay characterized the sheared profile. The $\beta$ was roughly $13 \sim 20^{\circ}$ in case of $\alpha^{\prime} / \alpha=30 / 64^{\circ}$, while the $\beta$ was roughly $18 \sim 22^{\circ}$ in case of $\alpha^{\prime} / \alpha=30 / 90^{\circ}$ by using the adhasives tape. Therefore, we can recognize that those cases were primary processed by using the secondary bevel angle $\alpha^{\prime}=30^{\circ}$, as shown in Fig. 8 .

Since there were the quasi-lower bound and the quasi-upper bound load response as a branching of deformation mode, the value of $\beta$ seemed to be affected by this mode branching. So that, in the case of $30 / 90^{\circ}$ facets blade, the peak cutting line force $f_{\text {peak }}$ and the inclined angle $\beta$ were arranged with respect to the combination of unwashed, washed surface condition and the feed velocities of $V=0.05,0.5 \mathrm{~mm} \cdot \mathrm{s}^{-1}$, as shown in Fig.13. 
Seeing the case of unwashed specimens, it is confirmed that the quasi-lower bound load response ( $f_{\text {peak }}$ was less than $80 \mathrm{kN} / \mathrm{m}$ or so) almost corresponded to $\beta<30^{\circ}$, while the quasi-upper bound load response was almost related to $\beta>30^{\circ}$. Here, the threshold of $30^{\circ}$ was assumed to be average of $\alpha^{\prime} / 2$ and $\alpha / 2$. Hence, it is revealed that the sheared profile is strongly affected by the branching of deformation mode (characterized by the quasi-upper/ quasi-lower bound load response). In the case of washed surface, the distribution of $\beta$ was not divided into any multiple zones. This is the reason why the dispersion of $\beta$ was smaller than that of unwashed condition.

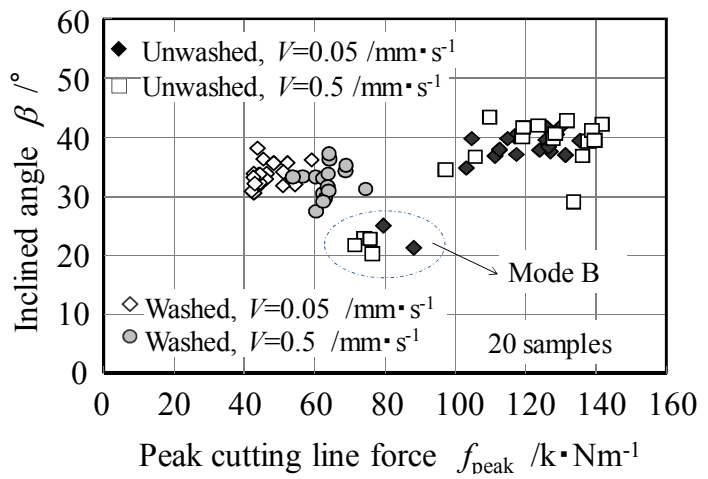

Fig.13 Relationship between peak cutting line force and inclined angle in case of $\alpha^{\prime} / \alpha=$ $30 / 90^{\circ}$ without adhesive tape

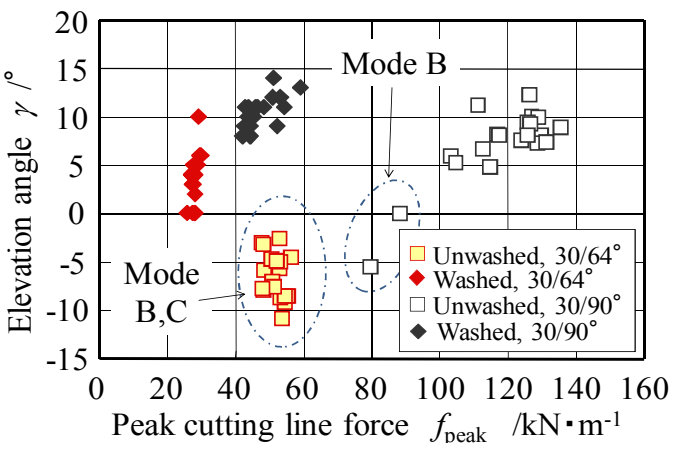

Fig.14 Relationship between peak cutting line force and elevation angle in case of $V=0.05$ $/ \mathrm{mm} \cdot \mathrm{s}^{-1}$ without adhesive tape

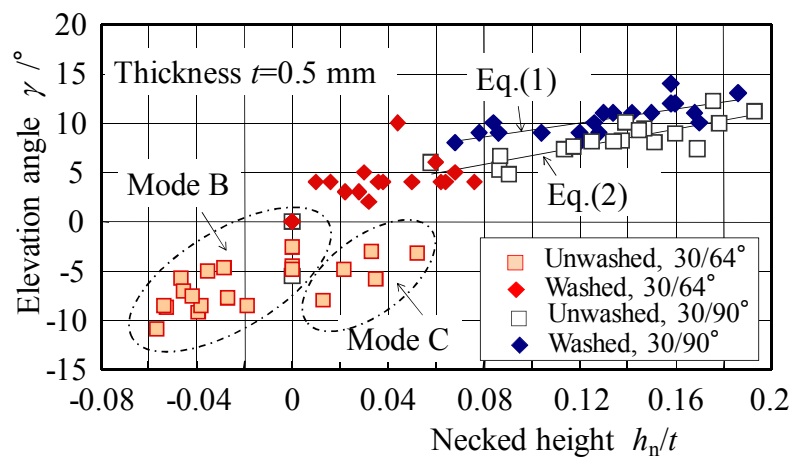

Fig. 15 Relationship between necked height and elevation angle in case of $V=0.05 / \mathrm{mm} \cdot \mathrm{s}^{-1}$ without adhesive tape

Regarding the branching of sheared profile, as mentioned above, we can understand that a pair of the upper-bound and the lower-bound load response corresponds to a pair of $\beta$ $>30^{\circ}$ and $\beta<30^{\circ}$ for $30 / 90^{\circ}$ facets blade. At the same time when $\beta$ was changed, other parameters $\gamma$ and $h_{\mathrm{n}}$ defined in Fig.11 were also investigated. Fig.14 shows the relationship between $f_{\text {peak }}$ and $\gamma$ in case of $V=0.05 \mathrm{~mm} \cdot \mathrm{s}^{-1}$ without adhesive tape $\left(\alpha^{\prime} / \alpha=30 / 64^{\circ}, 30 / 90^{\circ}\right)$. 
From this figure, it is found that the B mode $(\gamma \leq 0)$ remarkably occurred at the unwashed (sticky) condition in both cases of $\alpha^{\prime} / \alpha=30 / 64^{\circ}, 30 / 90^{\circ}$. The necked height $h_{\mathrm{n}}$ had varied without respect to $f_{\text {peak }}$, only except for unwashed condition with $\alpha^{\prime} / \alpha=30 / 90^{\circ}$. Therefore, instead of watching $f_{\text {peak }}$, the correlation between the $h_{\mathrm{n}} / t$ and the $\gamma$ was drawn in Fig.15 for $\alpha^{\prime} / \alpha=30 / 64^{\circ}, 30 / 90^{\circ}$ without adhesive tape $\left(V=0.05 \mathrm{~mm} \cdot \mathrm{s}^{-1}\right)$. It is evident that there is a positive correlation between $\gamma$ and $h_{\mathrm{n}} / t$. In case of $\alpha^{\prime} / \alpha=30 / 64^{\circ}$, the parameters $\gamma$ and $h_{\mathrm{n}} / t$ remarkably branched in two zones: $\mathrm{C}\left(\gamma<0, h_{\mathrm{n}} / t>0\right)$ or $\mathrm{B}\left(\gamma<0, h_{\mathrm{n}} / t<0\right)$, while $\beta$ was stably almost $15^{\circ}$ for all the experiments. As mentioned above for Fig.12, the primary reason of $\beta$ being $15^{\circ}$ is supposed to be caused by the secondary wedge of $\alpha^{\prime}=30^{\circ}$. In Fig. 15 , the mode $\mathrm{C}$ of $\alpha^{\prime} / \alpha=30 / 64^{\circ}$ was corresponded to the quasi-lower bound load response (e.g., Fig.5(a), $n_{\mathrm{C}}=7$ ), while the mode $\mathrm{B}$ of $\alpha^{\prime} / \alpha=30 / 64^{\circ}$ was corresponded to the quasi-upper bound load response (e.g., Fig.5(a), $n_{\mathrm{C}}=9$ ). According to video movies, the mode $\mathrm{B}$ was caused by a local sticking on the blade tip at the final breaking stage, while the mode $\mathrm{C}$ was a necking being caused by a lateral (In-Plane) sliding of the upper PC sheet. Namely, $h_{\mathrm{n}} / t$ is increased with In-plane necking deformation. Here, the bent-up angle of the upper PC sheet was quite trivial at the final breaking stage in case of $\alpha^{\prime} / \alpha=30 / 64^{\circ}$.

In the case of $\alpha^{\prime} / \alpha=30 / 90^{\circ}$, the mode B was corresponded to the quasi-lower bound load response (e.g., Fig.3(a), $n_{\mathrm{C}}=11$ ). Considering the contact pressure on the blade tip, we can confirm that the quasi-lower bound load with $\alpha^{\prime} / \alpha=30 / 90^{\circ}$ was close to the quasi-upper bound load with $\alpha^{\prime} / \alpha=30 / 64^{\circ}$. From Fig. 14 , the value of $f_{\text {peak }}$ was nearly 50 $\mathrm{kN} \cdot \mathrm{m}^{-1}$ for $\alpha^{\prime} / \alpha=30 / 64^{\circ}$, while it was nearly $80 \mathrm{kN} \cdot \mathrm{m}^{-1}$ for $\alpha^{\prime} / \alpha=30 / 90^{\circ}$. Since the projection width of the first facet is estimated as $2 h \cdot \tan (\alpha / 2)$, the mean pressure on the projection width was calculated as roughly $267 \mathrm{MPa}$ for the both cases.

In case of $\alpha^{\prime} / \alpha=30 / 90^{\circ}$, since the relationship between $\gamma$ and $h_{\mathrm{n}} / t$ had a keen-positive correlation in the mode A, the following normal equations (1),(2) were derived by using the linear least square method:

$$
\begin{array}{ll}
\gamma=36.2\left(h_{\mathrm{n}} / t\right)+5.67 & \text { (for washed) } \\
\gamma=44.6\left(h_{\mathrm{n}} / t\right)+2.28 & \text { (for unwashed) }
\end{array}
$$

As the mode A with $\alpha^{\prime} / \alpha=30 / 90^{\circ}$ occurred at a high pressure condition, the bent-up angle of the upper PC sheet was relatively large, as shown in Fig.2(a). Namely, $h_{\mathrm{n}} / t$ was increased with the bent-up deformation of the upper PC sheet. In this case, the necked end is forced by the bent-up deformation to rotate itself so as to increase the elevation angle $\gamma$.

So far, although the washed (slippery) condition with $\alpha^{\prime} / \alpha=30 / 64^{\circ}$ and the unwashed (sticky) condition with $\alpha^{\prime} / \alpha=30 / 90^{\circ}$ belonged to the mode A, those two conditions are generated from different mechanisms.

\section{Conclusions}

The indentation of a $30 / 90^{\circ}$ or $30 / 64^{\circ}$ facets (two-line) blade to a piled-up polycarbonate (PC) sheet of $t=0.5 \mathrm{~mm}$ thickness was experimentally carried out by varying the feed velocity and comparing washing surface condition (adhesive effect) of specimens in order to investigate the effect of contact surface adhesion and the sheared profile of PC sheet. In this work, the height of first bevel was chosen as $h / t=0.3$. Regarding the friction state on the contact surface of PC sheet, unwashed surface (sticky state), washed surface (slippery state) and lamination with adhesive tape were considered. The deformation flow in the side view of piled-up PC sheet subjected to a pushing load was observed by CCD microscope. Through this experiment, features of the cutting characteristic of piled-up structure were revealed as follows:

(1) When a certain glue layer of masking is coated on the PC sheet, the cutting load response (the relationship between the cutting line force $f$ and the indentation displacement of blade $c / t$ ) of facets blades is statistically varied with a certain state of dispersion before cutting off the upper PC sheet. 
(2) When the indentation displacement of blade $c / t$ is increased up to $0.3 \sim 0.6$, a lateral elongation of the upper PC sheet starts and after that time the upper PC sheet is apt to be bent up.

(3) For the latter half of $c / t>0.5$, there is a branching of deformation mode of the upper PC sheet. This branching was experimentally composed of two modes: the quasi-upper bound load response and the quasi-lower bound load response. In case of the former, there is the invariant area (dead zone) beneath the blade tip (first bevel) and also the underlay (lower PC sheet) begins upsetting when the cutting line force reaches at a certain level. In case of the latter, the first bevel successfully sinks to the upper PC sheet. As the result, the underlay is kept as the uniform body and the in-plane necking of the upper PC sheet is accelerated.

(4) By measuring the inclined angle $\beta$, the elevation angle $\gamma$ and the necked height $h_{\mathrm{n}}$ of the upper PC sheet, the sheared profile was statistically classified with three modes. Those modes are characterized with the bent-up angle of the upper PC sheet, the contact pressure beneath the first bevel blade, the friction condition of the PC sheets and the apex angle of the first bevel.

(5) In case of using adhesive tape, the dispersion of cutting load response is reduced. Through the experiment, asymmetric contact deformation on the center bevel blade was found.

(6) The feed velocity affected to the average of peak cutting line force $f_{\text {peak }}$ but not clearly to the statistical dispersion of $f_{\text {peak }}$.

\section{References}

(1) Hesse,F. \& Tenzer, H.J., Grundlagen der Papier-verarbeitung, VEB Verlag fur Buch und Bibliothekswesen, (1963), pp.58-60, Leipzig.

(2) Mizuguchi,S., Advanced Technology on Industrial Packaging and Transportation, Fuji Techno System, (2002), pp.58-59, Tokyo.

(3) Inaba,Y., Flatbed Diecutting and Maintenance of Die cutter, CARTON BOX, Vol.17 No.200 (1998), pp.17-20.

(4) Grunzweig,J., Longman,I. and Petch,N, Calculations and measurements on wedge-indentation, Journal of Mechanics and Physics of Solids, Vol.2, (1954), pp.81-86.

(5) Hill, R., On the Mechanics of Cutting Metal Strips with Knife-edged Tools, Journal of Mechanics and Physics of Solids, Vol.1, (1953), pp.265-270.

(6) Tamura,K., Effect of Fibrous Structure and Impurities on Fracture Phenomenon in Cutting Copper Strip between Knife-Edged Tools, SOSEI-TO-KAKOU, Vol.13 No.135 (1972), pp.242-250.

(7) Murayama,M., Nagasawa,S., Fukuzawa,Y. \& Katayama,I., Cutting Mechanism and Load Characteristic of Trapezoidal Center Bevel Cutter Indented on Aluminum Sheet, International Journal of Japan Society of Mechanical Engineers, C, Vol.47 No.1, (2004), pp.21-28.

(8) Chaijit,S., Nagasawa,S., Fukuzawa,Y., Murayama,M. \& Katayama,I., Effect of Tip Profile on Cutting Processability of Trapezoidal Cutting Blade Indented to Aluminum Sheet, Journal of Mechanics of Materials and Structures, Vol.1 No.8, (2006), pp.1301 $-1321$.

(9) Masumori,H., Hojo,H. \& Kageyama,Y., Effect of Shearing Speed and Temperature on Shearing Characteristics of Thermoplastic Polymer Sheets, SOSEI-TO-KAKOU, Vol.10 No.98, (1969), pp.180-189.

(10) Maeda,T. \& Mekaku,S. \& Makinouchi,A., Fine Cutting of Plastic Sheet with Knife-Edged Tool, SOSEI-TO-KAKOU, Vol.11 No.115, (1970), pp.617-624.

(11) Nagasawa,S., Murayama,M., Fukuzawa,Y. \& Sadamoto,A., Mechanics of Die Cutting for Paperboard Materials Processing, Kameda Book Service, (2004), pp.87-111, Niigata. 